\title{
Eingegangene Bücher zur Rezension
}

\section{Analytische Psychologie}

Wir freuen uns, wenn die Leserinnen und Leser der Zeitschrift sich an der Besprechung von Büchern beteiligen. Sie können zur Rezension bei der Redaktion angefordert werden. Nach dem Eintreffen des Buches bitte die Rezensionen in zweifacher Ausfertigung und auf Diskette (Windows/Word) oder per E-Mail spätestens innerhalb von 2 Monaten an die Redaktionsadresse senden.

Bergmann A: Ich und Du. Die Individuations- und Separationstheorie in psychoanalytischer Forschung und Praxis. Klett-Cotta, 2001.

Münch W: Märchenbilder und ihre Geheimnisse. Brandes \& Apsel, 2001.

Braun-Scharm H: Depressionen und komorbide Störungen bei Kindern und Jugendlichen. WVG, 2002.

Cabot Reid J: Jung, My Mother and I. The Analytic Diaries of C.R. Cabot. Daimon, 2001.

Claret BJ, Funke D, Schnock D, Schlagheck M (Hrsg): Theologie und Psychologie im Dialog über das Böse. Bonifatius, 1998.

Dammann G, Janssen P: Psychotherapie der Borderline-Störungen. Thieme, 2002.

Ethnopsychoanalyse Bd. 6: Forschen, erzählen und reflektieren. Brandes \& Apsel, 2001.

Fürstenau P: Psychoanalytisch verstehen - Systemisch denken - Suggestiv intervenieren. Pfeiffer, 2001.

Hauke C, Alister I (Hrsg): Jung and Film. Post-Jungian Takes on the Moving Image. Routledge, 2001.

Jaffe A: An Archetypal Approach to Death Dreams and Ghosts. Daimon, 1999.

Krumenacker F-J: Bruno Bettelheim. UTB-Reinhardt, 1998.

McNamee G (Hrsg): The Girl Who Made Stars and Other Bushman Stories. Daimon, 2001.

Mathers D: An Introduction to Meaning and Purpose in Analytical Psychology. Routledge, 2001.

Mayr U (Hrsg): Wenn Therapien nicht helfen. Pfeiffer, 2001.

Meier CA (Hrsg): Atom and Archetype. The Pauli/Jung Letters 1932-1958. Routledge, 2001.

Münnix N, Münnix G: Leben statt gelebt zu werden. Wie wir Kindern Orientierung geben. Walter, 1998.

Reddemann L: Imagination als heilsame Kraft. Zur Behandlung von Traumafolgen mit ressourceorientierten Verfahren. Pfeiffer, 2001.

Renggli F: Der Ursprung der Angst. Antike Mythen und das Trauma der Geburt. Walter, 2001.

Ribi A: Zeitenwende. Lang, 2001.

Sedgwick D: Introduction to Jungian Psychotherapy. Routledge, 2001.

Slattery DP, Corbett L (Hrsg): Depth Psychology: Meditations in the Field. Daimon, 2000.

Ulanov BA: The Wisdom of the Psyche. Daimon, 2000.

Wolff M (Hrsg): Selbst, Objekt und der Grundkonflikt. Brandes \& Apsel, 2001.

Zoja L, Romano ME: Drugs, Addiction and Initiation: The Modern Search for Ritual. Daimon, 1989.

\section{KARGER}

@2002 S. Karger AG, Basel

Fax +41613061234

E-Mail karger@karger.ch Accessible online at:

www.karger.com www.karger.com/journals/aps 